Brief report

\title{
Cortico-subcortical underpinnings of narrative processing impairment in schizophrenia
}

\author{
Gianfranco Spalletta ${ }^{\mathrm{a}, \mathrm{b}}$, Ilaria Spoletini ${ }^{\mathrm{a}}$, Andrea Cherubini ${ }^{\mathrm{a}}$, Ivo Alex Rubino ${ }^{\mathrm{b}}$, Alberto Siracusano ${ }^{\mathrm{b}}$, \\ Fabrizio Piras ${ }^{\mathrm{a}}$, Carlo Caltagirone ${ }^{\mathrm{a}, \mathrm{b}}$, Andrea Marini ${ }^{\mathrm{a}, \mathrm{c}, *}$ \\ a IRCCS Santa Lucia Foundation, Rome, Italy \\ ${ }^{\mathrm{b}}$ Department of Neuroscience, University of Rome "Tor Vergata", Rome, Italy \\ ${ }^{\mathrm{c}}$ University of Udine, Udine, Italy
}

\section{A R T I C L E I N F O}

\section{Article history:}

Received 12 June 2009

Received in revised form 30 October 2009

Accepted 2 November 2009

\section{Keywords:}

Schizophrenia

Language, narrative

Gray matter volumes

MRI

\begin{abstract}
A B S T R A C T
Cortical and subcortical gray matter volumes were correlated with a set of linguistic scores in a group of schizophrenia patients. Lexical informativeness was positively associated with the volume of the left frontal cortical and accumbal areas, while left hippocampal atrophy and right ventricle enlargement predicted increased production of semantic paraphasias. Global coherence impairment was predicted by left accumbal volume reduction and left ventricle enlargement. These data confirm that the brain structure of specific cortical and subcortal areas, as determined by magnetic resonance imaging, is related to the compromised semantic retrieval and language control in schizophrenia.
\end{abstract}

(c) 2009 Elsevier Ireland Ltd. All rights reserved.

\section{Introduction}

Language disturbance is one of the main clinical features of schizophrenia. Our group recently demonstrated that patients with schizophrenia have relatively disturbed lexico-semantic processing and are less informative and more tangential than healthy controls (Marini et al., 2008). There is evidence that patients with schizophrenia are characterized by structural abnormalities in subcortical brain areas, such as the hippocampus (Kalus et al., 2004), amygdala (Meisenzahl et al., 2008), thalamus (Rose et al., 2006), caudate, putamen, and accumbens (Spoletini et al., 2009), as well as in cortical areas such as fronto-temporal regions (Honea et al, 2005). These structures have also been reported as playing a crucial role in language processing (e.g. Crosson, 1997). Thus, the main purpose of the present study was to investigate the involvement of cortical and subcortical gray matter brain areas in language processing in schizophrenia.

\section{Methods}

\subsection{Participants}

Twenty-three Italian-speaking right-handed patients with diagnosis of schizophrenia were included in this investigation (see

\footnotetext{
* Corresponding author. Cattedra di Psicologia del Linguaggio, Università di Udine, Via Margreth, 3 - 33100 Udine (Italy). Tel.: +39 3355393224.

E-mail address: andrea.marini@uniud.it (A. Marini).
}

Table 1). These patients were a subsample of a larger cohort of 29 participants previously recruited for a linguistic study (Marini et al., 2008) who agreed to undergo a neuroimaging assessment. Detailed information on the study procedure is described elsewhere (Marini et al., 2008). All patients were in the phase of clinical stability and had been receiving stable oral doses of atypical antipsychotics for at least 1 month. Exclusion criteria included: substance abuse or dependence during the foregoing year; history of traumatic brain injury or any other neurological illness; any past or present major medical illness that may affect brain structures such as diabetes and cerebrovascular disease; any brain pathology identified on T2 or FLAIR scans; and mental retardation. Furthermore, in order to avoid the inclusion of patients with global cognitive deterioration, only patients scoring at least 23 on the Mini Mental State Examination (Folstein et al., 1975) were included. The study was approved by our local Ethics Committee and written consent was obtained from all participants after a full explanation of the procedures of the study.

\subsection{Narrative assessment}

Each patient described a single-picture image ("picnic", cf. Kertesz, 1982) and two cartoon stories with six pictures each ("flower pot", cf. Huber and Gleber (1982); "quarrel”, cfr. Nicholas and Brookshire (1993)). The modalities of the narrative assessment are described in Marini et al. (2008) and Marini et al. (2005). Here, we focused on three linguistic measures found to be strongly impaired in schizophrenia patients (i.e. semantic paraphasias, lexical informativeness and global coherence errors). These linguistic scores were calculated 
Table 1

Sociodemographic and clinical characteristics of the 23 patients with schizophrenia diagnosis.

\begin{tabular}{ll}
\hline Characteristics & $\begin{array}{l}\text { Schizophrenia patients } \\
(n=23)\end{array}$ \\
\cline { 2 - 2 } & Mean \pm SD \\
\hline Age (years) & $41.65 \pm 13.7$ \\
Educational level (years) & $11.1 \pm 3.2$ \\
Age at the onset of the illness (years) & $26.3 \pm 7.9$ \\
Duration of illness (years) & $15.3 \pm 11.7$ \\
PANSS Positive symptoms & $24.3 \pm 4.9$ \\
PANSS Negative symptoms & $20.7 \pm 7.2$ \\
PANSS General psychopathology & $50.0 \pm 10.5$ \\
Gender (male): $N$ (\%) & $16(69.6)$ \\
\hline
\end{tabular}

considering the mean values of percentages extracted from the three elicited stories.

\subsection{Image acquisition}

All participants underwent the same magnetic resonance imaging (MRI) protocol including standard clinical sequences (FLAIR, DP-T2weighted) and a high resolution whole-brain T1-weighted scan using a 3 T Allegra MR imager. Whole-brain T1-weighted images were obtained in the sagittal plane using a modified driven equilibrium Fourier transform (MDEFT) sequence (TE/TR $=2.4 / 7.92 \mathrm{~ms}$, flip angle 15 , voxel-size $\left.1 \times 1 \times 1 \mathrm{~mm}^{3}\right)$.

\subsection{Image processing and analyses}

\subsubsection{Subcortical gray matter}

Anatomical T1-weighted images were processed with the segmentation tool FIRST 1.1 integrated within the FSL software (Smith et al., 2004; http://www.fmrib.ox.ac.uk/fsl/). FIRST uses mesh models trained with a large amount of rich hand-segmented training data to segment subcortical structures. This method of segmentation is particularly useful for structures with low contrast-to-noise ratio. In each subject the lateral ventricles, caudate, thalamus, hippocampus, putamen, accumbens, pallidum, and amygdala were segmented. The segmentation results were visually assessed by two radiologists, and the volumes of the eight above-mentioned segmented subcortical areas were calculated. In order to reduce the effects of inter-individual variability in head size, individual volume values were multiplied by a normalization factor obtained with the SIENAX tool (http://www.fmrib.ox.ac.uk/fsl/siena/) from the corresponding T1-weighted image.

To assess which volume of subcortical brain areas predicted the linguistic performances (considered as dependent variables), we used a series of stepwise multiple regression analyses, with a forward procedure and an $F$ to enter of 4 . Pre-selection of subcortical area volume values to be included as independent variables in the stepwise regression models was performed by means of correlation analyses (Pearson's $r$ ) and Fisher's $r$ to $z$ transformation. In the stepwise multivariate models, only variables with $p<0.05$ in the pre-selection analyses were included.

\subsubsection{Cortical gray matter}

We applied voxel-based morphometry (VBM) to investigate regional cortical volume alterations in association with the linguistic variables, using statistical parametric mapping 5 (SPM5; Wellcome Department of Imaging Neuroscience, London, UK), running in Matlab 2007b (MathWorks, Natick, MA, USA). The T1-weighted images were normalized and segmented into gray and white matter partitions in the new unified segmentation step (Ashburner and Friston, 2005). The images were resliced with $1 \times 1 \times 1 \mathrm{~mm}^{3}$ voxels. The modulated and normalized gray matter images were smoothed with a Gaussian kernel of 12-mm full width at half maximum, on which all analyses were performed. To identify the brain regions wherein the patients showed volume changes correlated with the linguistic scores, three separate multiple regression models were undertaken, using lexical informativeness, semantic paraphasias and global coherence as regressors and age and education as covariates of no interest in the analyses (FWE $p<0.05$ corrected).

\section{Results}

\subsection{Subcortical gray matter}

Results of crude correlation analyses between linguistic performances and subcortical brain area volumes are shown separately for the three linguistic variables in Table 2 .

The only significant predictor of percentage of lexical informativeness was left accumbens volume. The resulting equation was significant $(F=10.825 ; d f=1,21 ; p=0.0035)$ and explained $34 \%$ $\left(r^{2}\right)$ of the overall variance of the lexical informativeness score. In particular, the lower left accumbal volume value predicted the reduced percentage of lexical informativeness (standard coefficient $=0.583$ ).

Significant predictors of percentage of semantic paraphasias were left hippocampus and right ventricle volumes. The resulting equation was significant $(F=28.518 ; d f=2.20 ; p=<0.0001)$ and explained $74 \%\left(r^{2}\right)$ of the variance of the semantic paraphasias score. In particular, a lower left hippocampus volume value predicted the increased percentage of semantic paraphasias $(F=13.254$; standard coefficient $=-0.498$ ), while a higher right ventricle volume value predicted the increased percentage of semantic paraphasias $(F=12.254$; standard coefficient $=0.479)$.

Significant predictors of percentage of global coherence errors were left accumbens and left ventricle volumes. The resulting equation was significant $(F=26.105 ; d f=2,20 ; p=<0.0001)$ and explained $74.1 \%\left(r^{2}\right)$ of the overall variance of the global coherence error score. In particular, lower left accumbal volume value predicted increased percentage of global coherence errors $(F=26.105$; standard coefficient $=-0.587$ ) and higher left ventricle volume value predicted an increased percentage of global coherence errors $(F=23.267$; standard coefficient $=0.554)$.

\section{Table 2}

Relationships between impaired linguistic performances and subcortical brain volume values in 23 patients with diagnosis of schizophrenia, as emerged from the univariate correlation analysis.

\begin{tabular}{|c|c|c|c|}
\hline & $\begin{array}{l}\text { \% lexical } \\
\text { informativeness }\end{array}$ & $\begin{array}{l}\% \text { global coherence } \\
\text { errors }\end{array}$ & $\begin{array}{l}\% \text { semantic } \\
\text { paraphasias }\end{array}$ \\
\hline & $\begin{array}{l}\text { Pearson's } \\
r(p \text {-value })\end{array}$ & $\begin{array}{l}\text { Pearson's } \\
r \text { ( } p \text {-value })\end{array}$ & $\begin{array}{l}\text { Pearson's } \\
r \text { ( } p \text {-value })\end{array}$ \\
\hline Right ventricle & $-0.309(0.1536)$ & $0.532(0.0080)$ & $0.754(<\mathbf{0 . 0 0 0 1})$ \\
\hline Left ventricle & $-0.224(0.3079)$ & $0.634(0.0008)$ & $0.692(\mathbf{0 . 0 0 0 1})$ \\
\hline Right thalamus & $0.229(0.2983)$ & $-0.120(0.5905)$ & $-0.562(\mathbf{0 . 0 0 4 4 )}$ \\
\hline Left thalamus & $0.122(0.5836)$ & $-0.034(0.8801)$ & $-0.373(0.0801)$ \\
\hline Right putamen & $0.281(0.1964)$ & $-0.353(0.0991)$ & $-0.432(\mathbf{0 . 0 3 8 8})$ \\
\hline Left putamen & $0.420(0.0455)$ & $-0.417(0.0472)$ & $-0.380(0.0735)$ \\
\hline Right hippocampus & $0.230(0.2958)$ & $-0.410(0.0513)$ & $-0.644(\mathbf{0 . 0 0 0 6})$ \\
\hline Left hippocampus & $0.331(0.1241)$ & $-0.405(0.0548)$ & $-0.762(<\mathbf{0 . 0 0 0 1})$ \\
\hline Right caudate & $0.245(0.2640)$ & $-0.316(0.1430)$ & $-0.334(0.1209)$ \\
\hline Left caudate & $0.209(0.3417)$ & $-0.284(0.1911)$ & $-0.356(0.0964)$ \\
\hline Right amygdala & $-0.214(0.3316)$ & $-0.060(0.7891)$ & $-0.082(0.7125)$ \\
\hline Left amygdala & $-0.125(0.5748)$ & $0.060(0.7882)$ & $0.353(0.0989)$ \\
\hline Right pallidum & $0.250(0.2534)$ & $-0.342(0.1110)$ & $-0.343(0.1104)$ \\
\hline Left pallidum & $0.377(0.0758)$ & $-0.397(0.0599)$ & $-0.339(0.1141)$ \\
\hline Right accumbens & $-0.031(0.8896)$ & $-0.104(0.6416)$ & $-0.376(0.0773)$ \\
\hline Left accumbens & $0.583(\mathbf{0 . 0 0 2 8})$ & $-0.662(\mathbf{0 . 0 0 0 4})$ & $-0.427(\mathbf{0 . 0 4 1 6})$ \\
\hline
\end{tabular}

Significant $p$-values $(p<0.05)$ are indicated in bold. 


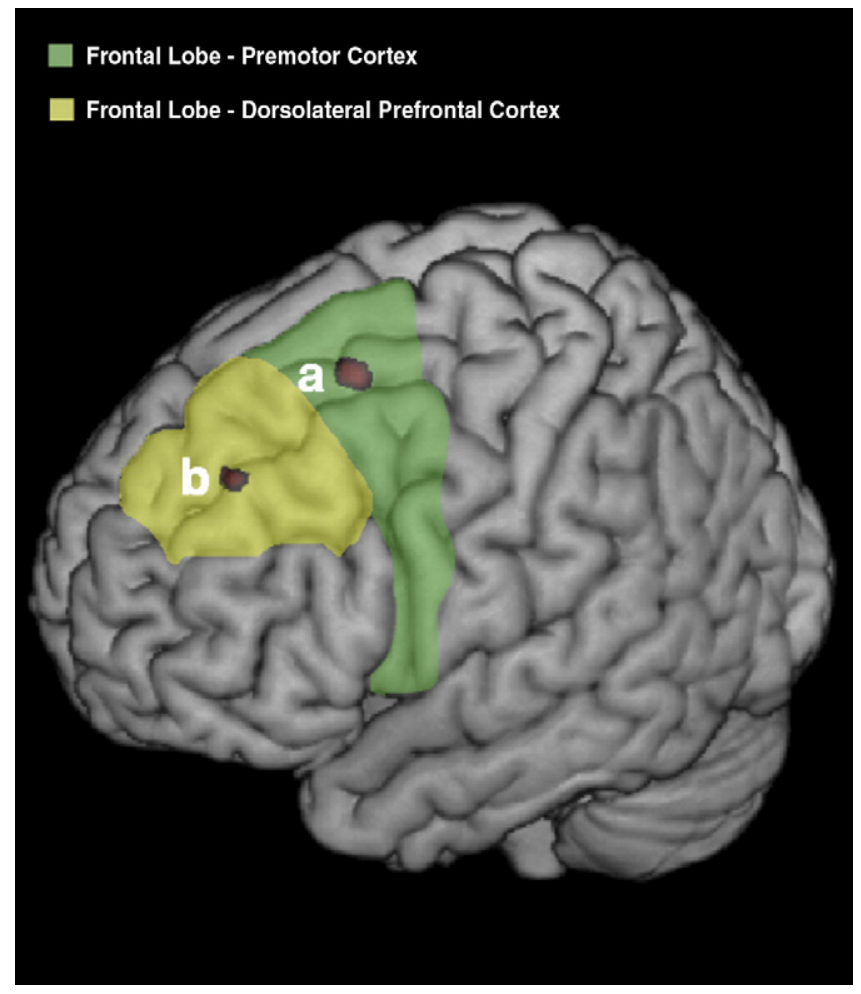

Fig. 1. Lateral view of the left hemisphere showing two frontal clusters of significant positive correlation between regional gray matter changes and lexical informativeness. Cluster a) MNI coordinates: -37 ; -11 ; 39; Brodmann's area: 6 ; cluster size: 90 voxels; voxel-level $t$-value: $6.14 ; z$-score: 4.55 ; FWE corrected cluster $p$-value: 0.032 . Cluster b) MNI coordinates: $-36 ; 18 ; 18$; Brodmann's area: 48 ; cluster size: 43 voxels; voxellevel $t$-value: 5.98 ; $z$-score: 4.48 ; FWE corrected cluster $p$-value: 0.041

\subsection{Cortical gray matter}

Two foci of volume changes in the left frontal cortex were found to significantly correlate with lexical informativeness (see Fig. 1). Specifically, an anterior cluster located in the prefrontal cortex (Montreal Neurological Institute (MNI) coordinates: - 36; 18; 18) and a more posterior one, located anteriorly to the primary motor cortex (MNI coordinates: $-37 ;-11 ; 39$ ). No correlations were found between regional gray matter changes and percentage of semantic paraphasias as well as errors of global coherence.

\section{Discussion}

Our results confirm the hypothesis of an involvement of cortical and subcortical structures in semantic retrieval and language control in schizophrenia. In particular, the production of semantic paraphasias is linked to decreased left hippocampal volume, suggesting the involvement of the hippocampus in at least some stages of the disrupted process of lexical selection in schizophrenia. Indeed, together with other medial temporal structures, the hippocampal area is a critical node in the network subserving declarative memory and is thought to be involved in the process of lexical retrieval (Buckner and Wheeler, 2001).

As for language control, global coherence maintenance is the ability to coherently link concepts in a story format by controlling what has been said, integrating the latter with incoming information and inhibiting irrelevant behaviours and the production of tangential utterances. A reduction in this high-level cognitive ability is linked in our sample study to decreased left accumbens volume and increased left lateral ventricle volume, which is an unspecific index of cortical atrophy. Interestingly, left accumbal volume, together with volume changes in selective areas of the left frontal cortex, also predicted the performance in lexical informativeness, which is the ability to choose linguistically and contextually appropriate lexical items. We explain these results in terms of the anatomical and functional meaning of the nucleus accumbens and its connections. The nucleus accumbens is a cell mass apposed to the rostroventromedial part of the caudateputamen complex, forming the ventral striatum, which is part of the basal ganglia (Nieuwenhuys et al 1988). The nucleus accumbens receives afferents from the subiculum, carrying the output from the hippocampal formation, the amygdala and the enthorinal cortex and receiving dopaminergic projections from the ventral tegmental area. Therefore, the accumbens represents the major site of integration between the limbic forebrain (hippocampal formation, amygdala, temporal and frontal cortices) and the basal ganglia. In line with the characteristics of its inputs, it has been found to be functionally associated with cognitive and motivational aspects of behaviour in schizophrenia (Gray, 1998). Consistently, our data suggest the involvement of left nucleus accumbens and left frontal areas in the disruption of high-level narrative-cognitive abilities in schizophrenia, i.e. lexical informativeness and global coherence. They are both related to the ability to select contextually appropriate lexical items and establish over-sentential links which maintain the selected items in working memory until necessary. Furthermore, they are also linked to the ability to control linguistic processing and eventually switch from an argument to the next. Therefore, as the two narrative abilities can be conceived of as a sort of language control, the involvement of the left nucleus accumbens may be explained as a component of a more complex circuit controlling linguistic behaviour, possibly involving the frontal cortical structures found in the present study. In this view, Crosson (1997) postulates a mainly inhibitory function of the striatum in regulating the release of preformulated language segments for motor programming and semantic verification. Dysfunction of these subcortical pathways can result in disinhibition of the speech areas in the frontal cortex, which furthermore implicates a loss of speech control. This is supported by the present findings showing that linguistic impairment in schizophrenia is related to volume changes in the nucleus accumbens (which is part of the striatum) and in two specific areas of the left frontal cortex that are thought to be involved in language motor programming (the premotor cortex, e.g. Fox et al., 2000) and in the selection of lexical information from competing alternatives (the dorsolateral prefrontal cortex, e.g. Nathaniel-James and Frith, 2002). Overall, this suggests that the ability of high linguistic load level is subserved by a distributed cortico-subcortical network which includes areas altered in their structures in patients with schizophrenia.

In conclusion, to the best of our knowledge, the results discussed here provide the first evidence of the involvement of left-lateralized cortical and subcortical volume changes (i.e. frontal, accumbal and hippocampal structures) in specific linguistic indexes previously found (Marini et al., 2008) to be impaired in patients with schizophrenia.

\section{References}

Ashburner, J., Friston, K.J., 2005. Unified segmentation. Neuroimage 26, 839-851. Buckner, R.L., Wheeler, M.E., 2001. The cognitive neuroscience of remembering. Nature Reviews. Neuroscience 2 (9), 624-634.

Crosson, B., 1997. Models of subcortical functions in language: current status. J. Neurolinguist. 10, 277-300.

Folstein, M.F., Folstein, S.E., McHugh, P.R., 1975, "Mini-mental state": a practical method for grading the cognitive state of patients for the clinician. Journal of Psychiatric Research 12 (3), 189-198.

Fox, P.T., Ingham, R.J., Ingham, J.C., Zamarripa, F., Xiong, J.H., Lancaster, J.L., 2000. Brain correlates of stuttering and syllable production. A PET performance-correlation analysis. Brain 123 (10), 1985-2004.

Gray, J.A., 1998. Integrating schizophrenia. Schizophr. Bull. 24 (2), 249-266.

Huber, W., Gleber, J., 1982. Linguistic and non-linguistic processing of narratives in aphasia. Brain and Language 16 (1), 1-18.

Kalus, P., Buri, C., Slotboom, J., Gralla, J., Remonda, L., Dierks, T., Strik, W.K., Schroth, G., Kiefer, C., 2004. Volumetry and diffusion tensor imaging of hippocampal subregions in schizophrenia. Neuroreport 15 (5), 867-871. 
Kertesz, A., 1982. The Western Aphasia Battery. Grune \& Stratton, New York.

Marini, A., Boewe, A., Caltagirone, C., Carlomagno, S., 2005. Assessment of age-related differences in the processing of textual descriptions. Journal of Psycholinguistic Research 34/5, 439-463.

Marini, A., Spoletini, I., Rubino, I.A., Ciuffa, M., Banfi, G., Siracusano, A., Bria, P., Caltagirone, C., Spalletta, G., 2008. The language of schizophrenia: an analysis of micro- and macrolinguistic abilities and their neuropsychological correlates. Schizophrenia Research 105 (1-3), 144-155.

Meisenzahl, E.M., Koutsouleris, N., Bottlender, R., Scheuerecker, J., Jäger, M., Teipel, S.J., Holzinger, S., Frodl, T., Preuss, U., Schmitt, G., Burgermeister, B., Reiser, M., Born, C., Möller, H.J., 2008. Structural brain alterations at different stages of schizophrenia: a voxel-based morphometric study. Schizophrenia Research 104 (1-3), 44-60.

Nathaniel-James, D.A., Frith, C.D., 2002. The role of the dorsolateral prefrontal cortex: evidence from the effects of contextual constraint in a sentence completion task. Neuroimage 6 (4), 1094-1102.

Nieuwenhuys, R., Voogd, J., van Huijzen, C., 1988. The Human Central Nervous System, third ed. Springer-Verlag, Berlin.
Nicholas, L.E., Brookshire, R.H., 1993. A system for quantifying the informativeness and efficiency of the connected speech of adults with aphasia. Journal of Speech and Hearing Research 36 (2), 338-350.

Honea, R., Crow, T.J., Passingham, D., Mackay, C.E., 2005. Regional deficits in brain volume in schizophrenia: a meta-analysis of voxel-based morphometry studies. American Journal of Psychiatry 162, 2233-2245.

Rose, S.E., Chalk, J.B., Janke, A.L., Strudwick, M.W., Windus, L.C., Hannah, D.E., McGrath, J.J. Pantelis, C., Wood, S.J., Mowry, B.J., 2006. Evidence of altered prefrontal-thalamic circuitry in schizophrenia: an optimized diffusion MRI study. Neuroimage 32 (1), 16-22.

Smith, S.M., Jenkinson, M., Woolrich, M.W., Beckmann, C.F., Behrens, T.E.J., Johansen-Berg, H., Bannister, P.R., De Luca, M., Drobnjak, I., Flitney, D.E., Niazy, R., Saunders, J., Vickers, J. Zhang, Y., De Stefano, N., Brady, J.M., Matthews, P.M., 2004. Advances in functional and structural MR image analysis and implementation as FSL. Neuroimage 23 (S1), 208-219. Spoletini, I., Cherubini, A., Banfi,G., Rubino, I.A., Peran, P., Caltagirone, C., Spalletta, G., 2009 Hippocampi, thalami and accumbens microstructural damage in schizophrenia: volumetry, diffusion, and neuropsychological study. Schizophrenia Bulletin, epublication 19 June 2009. doi:10.1093/schbul/sbp058. 